\title{
An IFPI Temperature Sensor Fabricated in an Unstriped Optical Fiber with Self-Strain-Compensation Function
}

\author{
Yang Song, ${ }^{1}$ Liwei Hua, ${ }^{1}$ Jincheng Lei, ${ }^{1}$ Qi Zhang, ${ }^{1}$ Jie Liu, ${ }^{1}$ Lingyun Ye, ${ }^{2}$ and Hai Xiao ${ }^{1}$ \\ ${ }^{1}$ The Holcombe Department of Electrical and Computer Engineering, Center for Optical Materials Science and \\ Engineering Technologies (COMSET), Clemson University, 105 Riggs Hall, Clemson, SC 29634, USA \\ ${ }^{2}$ Zhejiang University, Yuquan Campus, Zheda Road 38th, Hangzhou, Zhejiang 310027, China \\ Correspondence should be addressed to Lingyun Ye; lyye@zju.edu.cn
}

Received 23 August 2016; Accepted 9 October 2016

Academic Editor: Banshi D. Gupta

Copyright ( 2016 Yang Song et al. This is an open access article distributed under the Creative Commons Attribution License, which permits unrestricted use, distribution, and reproduction in any medium, provided the original work is properly cited.

\begin{abstract}
This paper describes an intrinsic Fabry-Perot interferometer (IFPI) temperature sensor with self-strain-compensation function. The sensor was fabricated on a buffer-intact optical fiber using a femtosecond ( $\mathrm{fs}$ ) laser system. The use of fs laser allows the sensor to be fabricated in an optical fiber without the necessity of removing the polymer buffer coating, thus not compromising its mechanical property. The sensor is composed of two cascaded IFPIs in different cavity length of $100 \mu \mathrm{m}$ and $500 \mu \mathrm{m}$, respectively. The shorter IFPI serves as the temperature sensor, while the second IFPI serves as a compensation sensor, which is used to decouple the strain from the raw signal collected by the shorter FPI. The reflection spectrum of sensor, containing both sensory information and compensation information, is collected in wavelength domain and demultiplexed in the Fourier domain of reflection spectrum. An algorithm was developed and successfully implemented to compensate the strain influence on the proposed temperature sensor. The results showed that the proposed sensor structure holds a constant temperature sensitivity of $11.33 \mathrm{pm} /{ }^{\circ} \mathrm{C}$ when strained differently.
\end{abstract}

\section{Introduction}

Optical fiber based sensors have been intensively studied for sensing various kinds of physical quantities, including temperature, strain, pressure, and bio/chemical quantities, like intracellular $\mathrm{pH}$, chemical concentration, and so forth. A variety of optical fiber sensor structures, as well as sensing mechanisms, was investigated in the past two decades $[1,2]$. A well-known example is Fabry-Perot (FP) interferometer, containing a pair of low-reflectivity mirrors to generate two reflection beams with differing phase delays. Thus, an interference pattern is formed in its reflection spectrum that can be used to accurately measure the phase delay between two mirrors for sensing applications. FPI sensors can be subcategorized into two types, extrinsic Fabry-Perot interferometer (EFPI) and IFPI. EFPI is able to facilitate direct wavematter interaction, while IFPIs are supreme in its advantages in stability, all-in-fiber flexibility, and lower insertion loss. Uniquely, the features of IFPIs are highly desired for harsh environment sensing.
In the past years, several types of distributed sensor with cascaded FPIs have been demonstrated. The major tracking methods are to demodulate the multiplexed interferometers by fast Fourier transform (FFT). A quasi-distributed IFPI sensing system demultiplexed with FFT-based wavelength tracking method was presented at early years [2]. The wavelength tracking method was utilized to demodulate the temperature information applied to all IFPIs. Frequency components corresponding to each sensor are extracted from the transmission spectra and then transform back to the wavelength domain [3]. The experimental results of the multiplexed IFPI sensors showed the high reliability of wavelength tracking methods according to the high temperature sensitivity. Later, a reported type of sensor used cascaded extrinsic FP interferometer (EFPI) and IFPI as temperature and pressure sensing elements [1]. The sensing system could measure temperature and pressure unambiguously and similarly testified the wavelength tracking methods. But, on the other hand, those sensors which detected single varying quantity and gave 
the analysis of sensitivity assumed no crosstalk from other changing parameters at the sensing area.

In this paper, a temperature sensor system with strain selfcompensation is presented. Dual-parameters sensing design using cascaded IFPI structures resolved the deviation from temperature-strain crosstalk. To achieve the high quality of optical structure inside fiber, femtosecond laser micromachining was employed in fabricating IFPIs. This ultrafast laser delivers high-intensity energy to the core of single-mode fiber (SMF) and effectively changes the refractive index in inscribed region. During the fabrication, the intact polymer buffer coating of SMF is not striped off, which significantly enhances the mechanical prosperity of the sensor [4]. A pair of IFPIs with different cavity lengths including the main sensor and the compensation sensor composes this sensor system.

\section{Demultiplex Principle}

IFPI is sensitive to temperature due to the combination of the thermooptic effect and thermal expansion of the fiber material. In addition, it is strain sensitive because of the variation of refractive index (RI) and the optical length changing simultaneously with physical length of the sensor changing under stretch. Strain-induced effect is defined by Young's modulus multiplexing the stress. Therefore, temperature and strain variation are independent variables to the IFPIs because temperature change will not significantly affect Young's modulus that could be ignored. Specifically, the laser ablated cavity mirrors reflect the guided light from laser source under the influence of temperature and strain $[5,6]$. Figure 1 is the structural schematic of IFPI. The two reflected lights formed both sides of the IFPI and denoted and generated an inference pattern. The equation of FP interferometer can be modeled by the two-beam optical interference equation

$$
I=I_{1}+I_{2}+2 \sqrt{I_{1} I_{2}} \cos \left(\frac{2 \pi \cdot \mathrm{OPD}}{\lambda}+\varphi_{0}\right),
$$

where $I$ is the intensity of the interference signal; $\varphi_{0}$ is the initial phase of the interference (normally equals zero); and $\lambda$ is the optical wavelength. The round-trip optical path difference (OPD) of the FP interferometer is given by

$$
\mathrm{OPD}=2 n_{\text {cavity }} L
$$

where $n_{\text {cavity }}$ is the RI of the cavity medium and $L$ is the physical cavity length of each IFPI. At the valleys of the interferogram in spectrum domain, the phase difference of the two light beams satisfies the condition of destructive interference:

$$
\frac{4 \pi n_{\text {cavity }} L}{\lambda_{m}}+\varphi_{0}=(2 m+1) \pi,
$$

where $m$ is an integer and $\lambda_{m}$ is the wavelength of the $m$ th order interference valley [7]. The distance between two adjacent minima of the spectrum, defined as the free spectrum range (FSR), can then be expressed as [8]

$$
\mathrm{FSR}=\frac{\lambda^{2}}{2 n_{\text {cavity }} L} .
$$

In this sensor system, the compensation IFPI sensor has wavelength shift as a function strain as

$$
\Delta \lambda_{\varepsilon}=\left(-P_{\mathrm{eff}}+1\right) \varepsilon \cdot \lambda_{m}
$$

where $P_{\text {eff }}$ is the effective strain-optic coefficient, approximately 0.204 for fused silica material [8]. From (5), the strain sensitivity is dependent on the interrogated wavelength and the effective strain-optic coefficient [8].

The wavelength shift caused by temperature variation around main IFPI sensor can be expressed as the following equation:

$$
\Delta \lambda_{T}=\left(\alpha_{\mathrm{CTE}}+\frac{d_{n}}{d_{T}}\right) \cdot \lambda_{m} \cdot \Delta T
$$

where $a_{\mathrm{CTE}}$ and $d_{n} / d_{T}$ are the thermal expansion coefficient and thermal-optic coefficient of the material, respectively. For fused silica material, the CTE is $0.55 \times 10^{-6} /{ }^{\circ} \mathrm{C}$ and the thermooptic coefficient is approximately $7 \times 10^{-6} /{ }^{\circ} \mathrm{C}[9,10]$. Based on (5) and (6), the temperature-strain crosstalk of the main IFPI sensor is given by

$$
\frac{\mu \varepsilon}{\Delta T}=\frac{\Delta \lambda_{T} / \Delta T}{\Delta \lambda_{\varepsilon} / \mu \varepsilon}=\frac{\left(\alpha_{\mathrm{CTE}}+d_{n} / d_{T}\right) \cdot 10^{6}}{-P_{\mathrm{eff}}+1} .
$$

The calculated result of the temperature-strain crosstalk for the main IFPI sensor is $9.57 \mu \varepsilon /{ }^{\circ} \mathrm{C}$ [1].

In general, the wavelength shift of the sensing system can be expressed as

$$
\begin{aligned}
\Delta \lambda_{\text {compensate }} & =K_{\varepsilon} \times \mu \varepsilon \\
\Delta \lambda_{\text {main }} & =K_{\varepsilon} \times \mu \varepsilon+K_{T} \times \Delta T,
\end{aligned}
$$

where $K_{\varepsilon}$ is the coefficient of strain-induced effect and $K_{T}$ is the coefficient of temperature-induced effect. The strain and temperature can be obtained by solving the following matrix:

$$
\left[\begin{array}{c}
\Delta T \\
\mu \varepsilon
\end{array}\right]=\frac{1}{A}\left(\begin{array}{cc}
K_{\varepsilon} & -K_{\varepsilon} \\
-K_{T} & 0
\end{array}\right)\left[\begin{array}{c}
\Delta \lambda_{\text {compensate }} \\
\Delta \lambda_{\text {main }}
\end{array}\right],
$$

where $A=-K_{\varepsilon} \cdot K_{T}$.

The interference signal of the sensing system is multiplexed. OPD of the two IFPIs can be resolved by performing the Fourier transform on the recorded interferogram. Broadband light source with a range from 1520 to 1620 with minimum detectable OPD change of $12 \mu \mathrm{m}$ is used in this paper. As early reported, a wavelength tracking method based on FFT is used to improve the measurement accuracy and demodulate the multiplexed signals [2]. Demodulation of the IFPIs spectrum signal follows these steps. First, the two IFPI free spectrum ranges can be distinguished in frequency domain through FFT. Then band-pass filters are used to extract two separate frequency components and do the inverse FFT. The obtained individual wavelength domain spectrum is utilized to track the wavelength shift for each IFPI. 


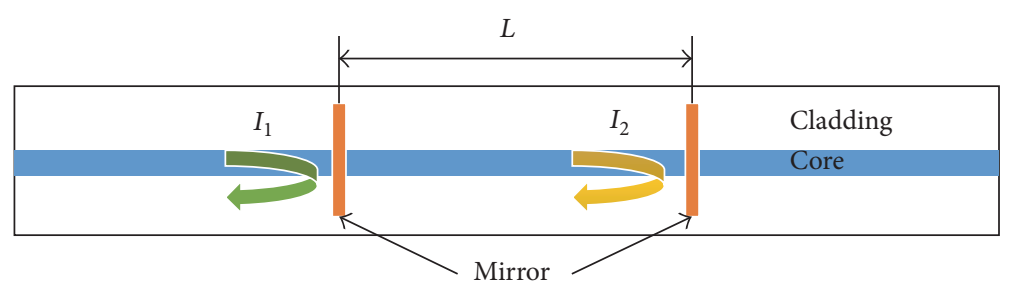

FIGURE 1: Structural schematic of IFPI.

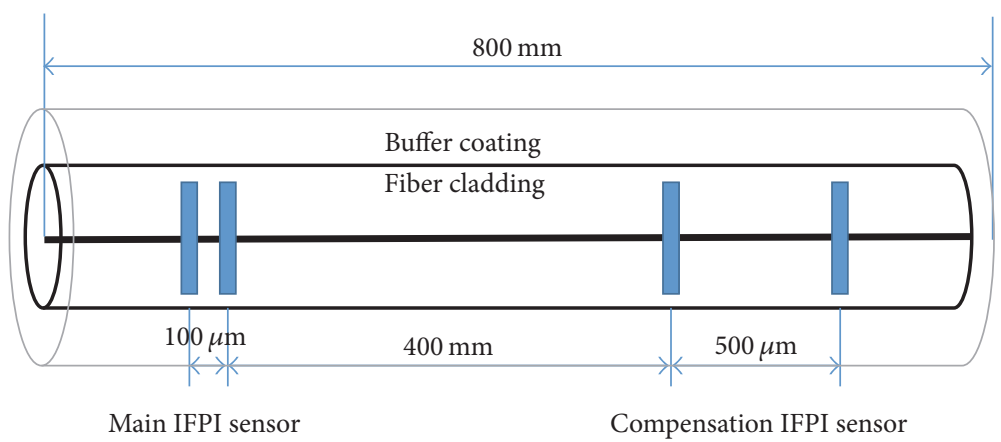

FIGURE 2: Cascaded IFPI sensor system structure.

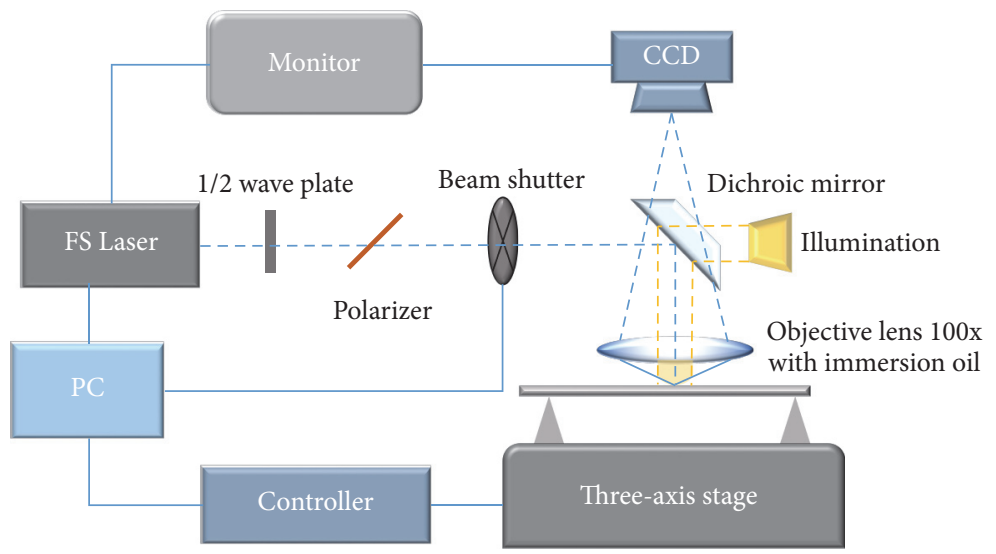

FIGURE 3: Schematic of the fs laser micromachining system.

\section{Sensor Design and Fabrication}

The sensor consists of two pairs of low reflectance cavity mirrors that form two cascaded IFPIs. Each reflector is created by laser micromachining in the core of a single-mode fiber (Corning SMF-28e). The cavity length of main IFPI sensor is $100 \mu \mathrm{m}$ and that of the so-called compensation one is $500 \mu \mathrm{m}$.

Figure 2 shows the sensor structure and dimensions. Four low reflectance mirrors are $15 \mu \mathrm{m}$ in width, $15 \mu \mathrm{m}$ in height, and $1 \mu \mathrm{m}$ in thickness. The distance between two IFPIs is $400 \mathrm{~mm}$ to ensure that temperature changes merely around main IFPI sensor. The total mounted length of fiber is $800 \mathrm{~mm}$. In practical application, the dimensions are flexible in design according to actual needs. As mentioned, the two IFPIs are both sensitive to temperature and strain.

In the case of the stripped-off fabrication, the bare fiber is lack of mechanical property and easy to break, not robust in experiment or any other harsh environments [4]. Pliability is reduced due to the crippled integrity even with recoating process. Therefore, the engineering challenge in this sensor is to keep the acrylate buffer coating during fabrication with no damage in the coating. Improper laser power and focal delivery will result in melting the polymer coating and defocusing the laser beam.

To assist the fabrication of IFPIs in SMF, FS laser micromachining is used [11]. Figure 3 shows the system of Ti: sapphire fs laser (Coherent Inc.) with the central wavelength in $800 \mathrm{~nm}$, pulse width in $200 \mathrm{fs}$, and repetition rate of the laser in $250 \mathrm{KHz}$, respectively. The maximum output power of the laser is $1 \mathrm{~W}$. The actual power used for fabrication was controlled by adjusting the laser beam optics, including a half-wave plate, a polarizer, and one linear neutral density filter. A beam shutter (Thorlabs) was used for switching the laser from on and off. The actual pulse energy for fabrication in this experiment is $0.32 \mu \mathrm{J}$. The fs laser beam was 


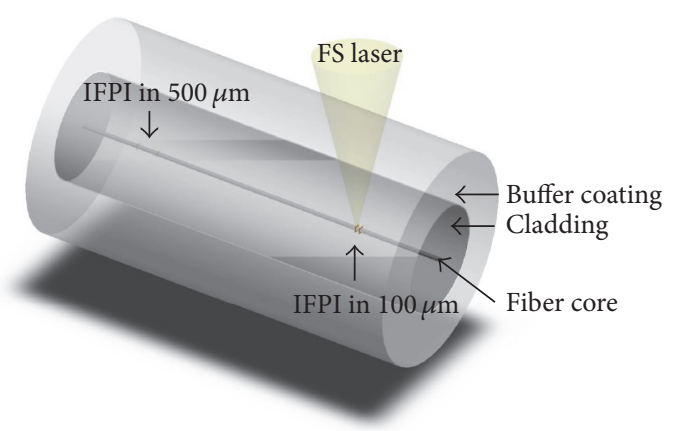

(a)

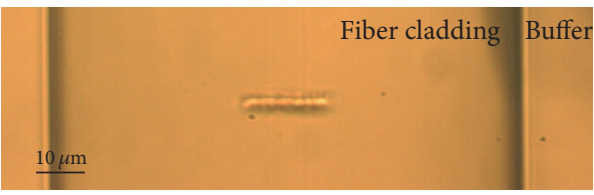

(b)

FIgURE 4: (a) Schematic of the fs laser fabrication. (b) Micrograph of the inscribed region.

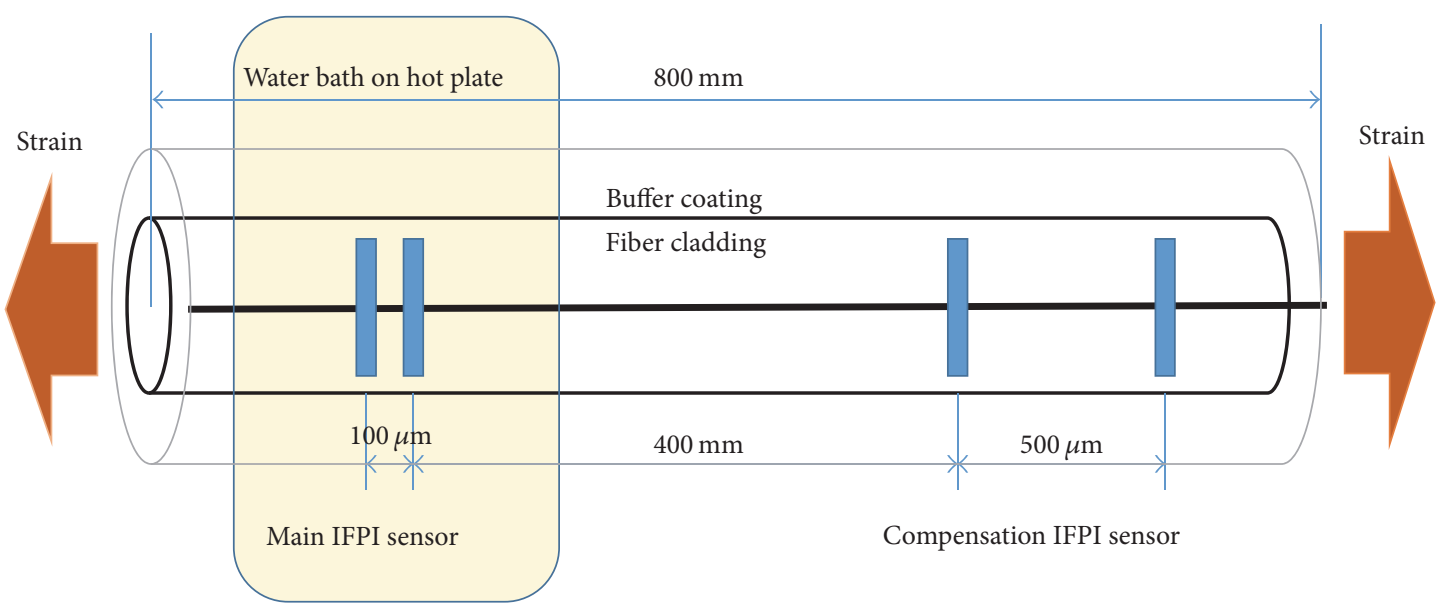

FIGURE 5: Schematic of the experiment setup.

focused into the fiber through an oil immersion objective lens (Olympus. UMPLanFX 100x) with a numerical aperture (NA) of 1.3 [7]. The whole cross-section of the fiber core is covered by a cuboid region of $15 * 1 * 15 \mu \mathrm{m}$ inscribed in the center of the fiber from the bottom up. The scanning speed is $20 \mu \mathrm{m} / \mathrm{s}$. Figure 4 shows the schematic diagram of the fabrication with buffer intact and the micrograph of the inscribed region which forms a low reflectance mirror.

Refractive index matching oil (Type A, Cargille) with low viscosity and refractive index of 1.46 is used as transparent immersion medium. In oil immersion, the aspect ratio value of the laser spot is higher than that in water immersion significantly. This ratio value depends on the NA and the NA value can be obtained only with oil/water immersion objectives where the lateral spatial resolution can be less than $100 \mathrm{~nm}$. The large NA due to the high refractive index of matching oil helps to improve the aspect ratio of the inscribed region. More importantly, it prevents the heat absorption and melting in the acrylate buffer coating more effectively.

\section{Experimental Result}

In experiment, the strain sensor system is mounted one side on a moto-driven one-axis stage (Newport) and the other side on a fixed post. The stage has axis resolution of $50 \mathrm{~nm}$ and it is controlled to move a certain length by its own software. Applied strain occurs when the stage move outwards until the fiber is tight. Figure 5 shows the experiment setup with strain and temperature environment. For the main IFPI sensor temperature test, a hotplate with thermal couple provides heating in water bath $30-90^{\circ} \mathrm{C}$ as the acyclic buffer coating will not survive at high temperature due to the low melting points. The distance between two IFPIs is $400 \mathrm{~mm}$ and total length of fixed optical fiber is $800 \mathrm{~mm}$, respectively. This cascaded sensor system is interrogated by a broadband light source. The wavelength range is from 1520 to $1620 \mathrm{~nm}$. The light is routed by a $3 \mathrm{~dB}$ coupler and the interference spectrum is detected by an optical spectrum analyzer (AQ6319).

Figure 6 shows the recorded spectrum of multiplexed sensing signal in air and the demodulated result after FFT. 


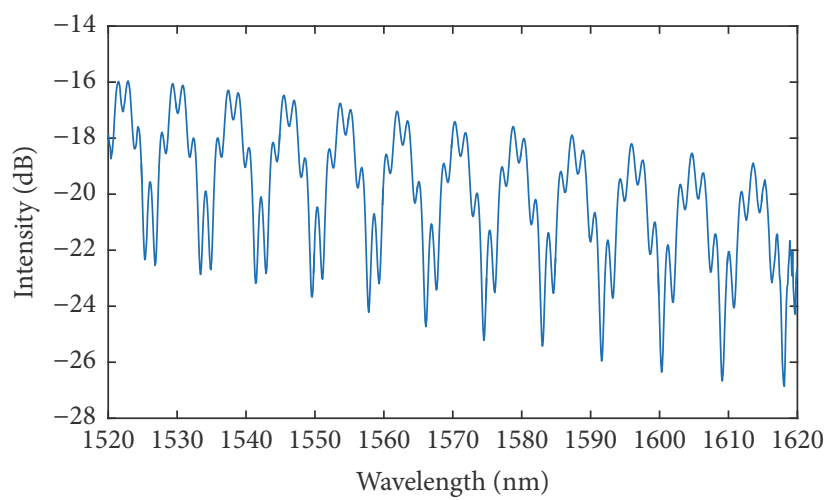

(a)

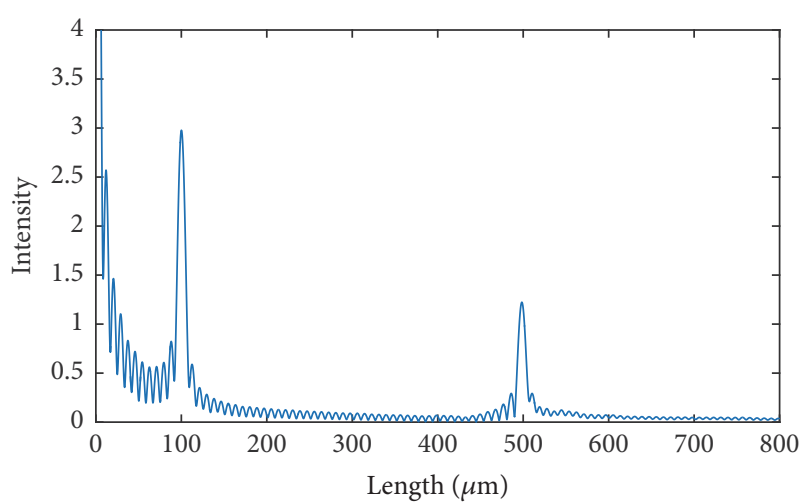

(b)

FIGURE 6: (a) Recorded spectrum of cascaded IFPI sensors. (b) FFT result of the multiplexed sensors.

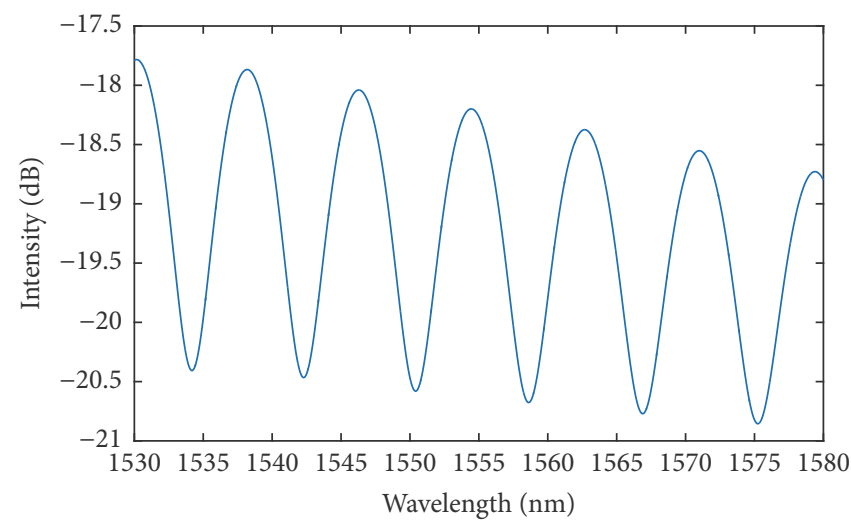

(a)

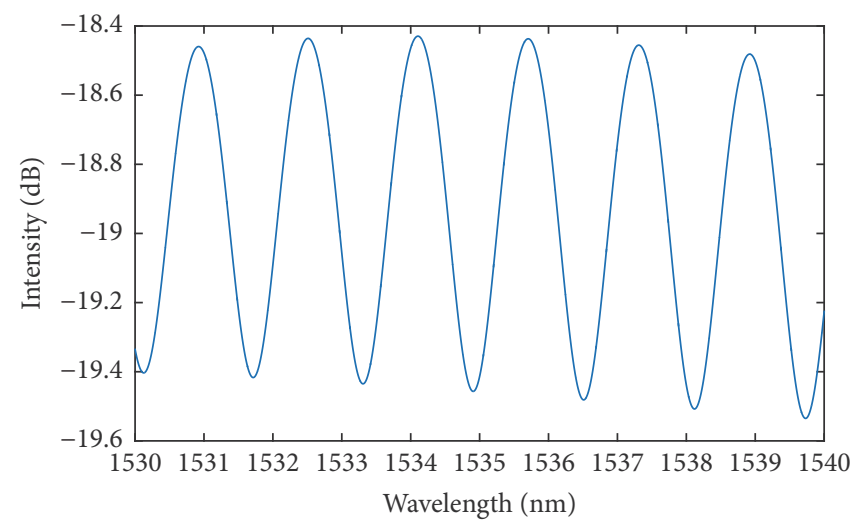

(b)

FIGURE 7: Reconstruction of wavelength signal of (a) main IFPI sensor in $100 \mu \mathrm{m}$ and (b) compensation IFPI sensor in $500 \mu \mathrm{m}$.

The waveform domain signal has a high fringe visibility of $7 \mathrm{~dB}$. Since the recorded signal is in wavelength axis and only the wave number axis spectrum is suitable for FFT, linspace function in MATLAB library is invoked to arrange the sampling points of wave number domain spectrum in equal interval. In Figure 6(b), two space frequency components with substantially different OPDs can be clearly identified. To select the individual frequency domain component, Hanning-windowed digital filters were used in FFT plotted result. Next, the filtered result is transferred back to the wavelength spectrum. Figures 7 (a) and 7 (b) are the reconstructed waveforms of two IFPIs, respectively. Once we have the individual interferogram of the two IFPIs, the tracking wavelength can be picked from a specific interference dip in the reconstructed spectrum. Further on, the waveform shift is obtained from this tracking method.

The first experiment is to calibrate the temperature response from main IFPI sensor. Water bath on hot plate (Corning) with thermal couple provides temperature change from 30 to $90^{\circ} \mathrm{C}$ with $10^{\circ} \mathrm{C}$ every step [12]. Fiber is loose in sensing area with no strain exerted. In theory, the compensation IFPI sensor will not give any wavelength shift.

Dynamic measurement result of the sensor system performs the specific function in temperature sensitive and strain self-compensation. In dual varying parameters, main IFPI sensor gives wavelength spectrum shifts from combined impact of temperature and strain. The temperature effect is refined by linearly subtracting the strain information from main sensor signal. Figure 8(a) shows a dip in reconstructed spectrum shifting from the compensation IFPI sensor between $1534 \mathrm{~nm}$ and $1536 \mathrm{~nm}$ and Figure 8(b) is the straininduced response. Initialized position of the strain text is set once the fiber is tight. According to the calculation of temperature-strain crosstalk in principle, strain from initialization to additional $600 \mu \varepsilon$ with $100 \mu \varepsilon$ per step is exerted. Simultaneously, temperature test on main IFPI sensor goes again. The number of sampling points in strain test matches the number in temperature test. In Figure 8(b), the compensation IFPI sensor spectrum shifts towards the longer wavelength under increasing strain linearly and the response slope is $1.17 \mathrm{pm} / \mu \varepsilon$.

For the main IFPI sensor, the waveform in Figure 9(a) shifts similarly to the longer wavelength range corresponding to dual parameters. Plotted result of linear relationships in Figure 9(b) shows the wavelength shift information from main IFPI sensor before and after the compensation. Straininduced effect is dislodged from recorded spectrum by linearly subtracting the spectrum in Figure 8(b) from the main result. The calibration of temperature response in main IFPI 


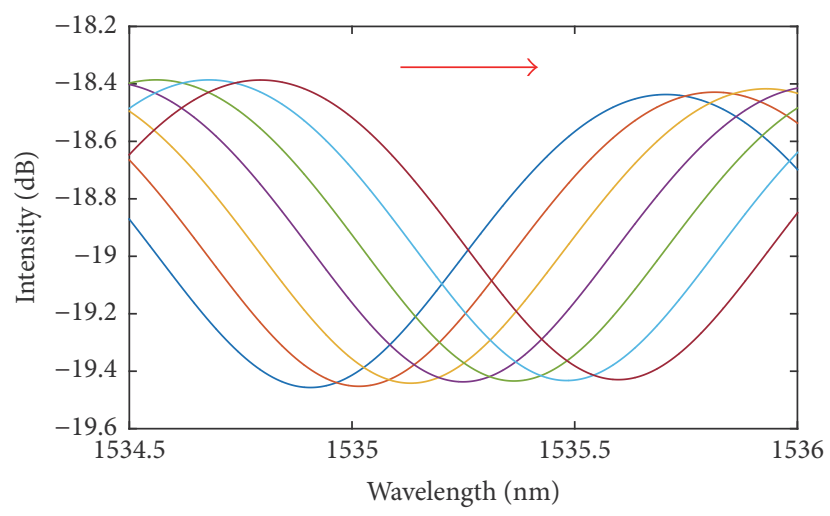

(a)

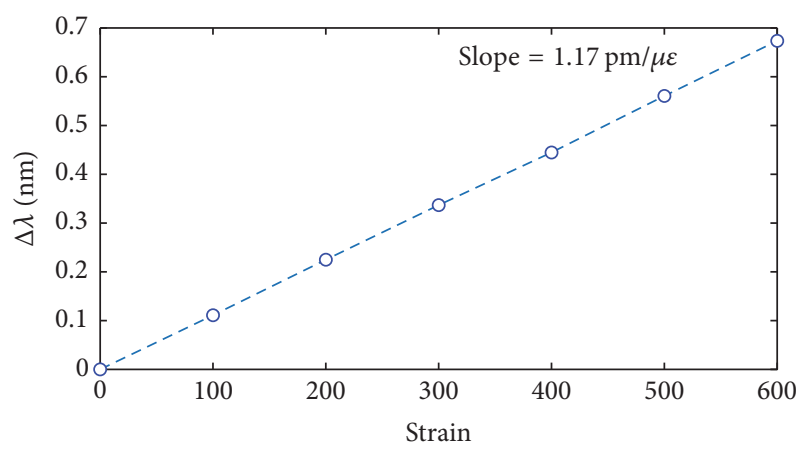

(b)

FIGURE 8: (a) Compensation IFPI sensor waveform shifts to the longer wavelength range. (b) Strain test result with slope in $1.17 \mathrm{pm} / \mu \varepsilon$.

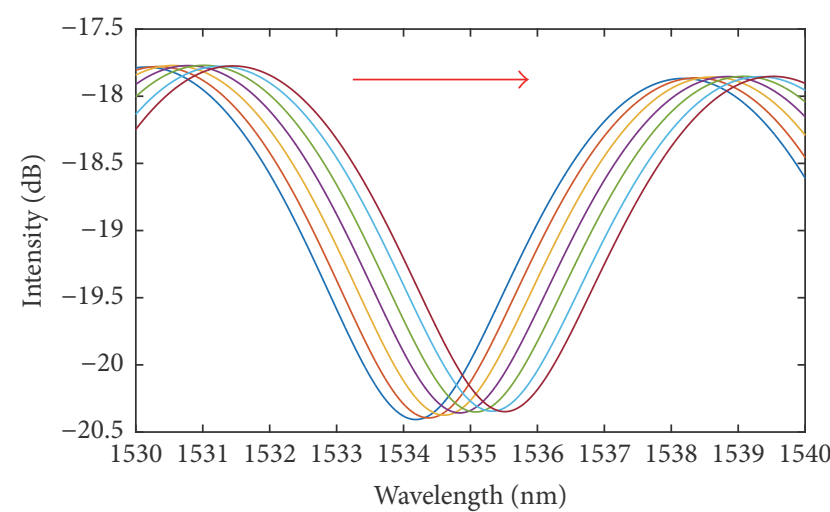

(a)

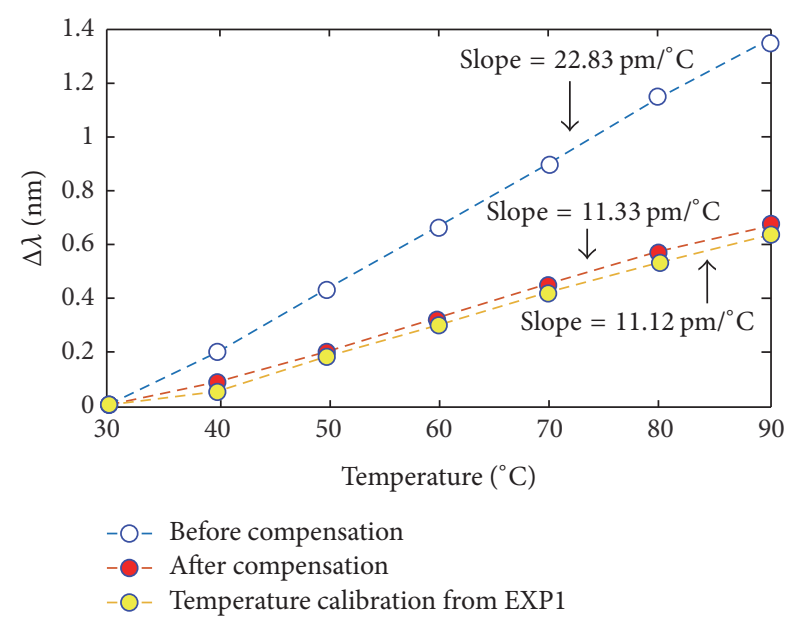

(b)

Figure 9: (a) Main IFPI sensor waveform shifts to the longer wavelength range. (b) Test result before compensation in blue line. After compensation, by linearly subtracting the strain information, the result of spectrum matches the temperature calibration from experiment 1 .

trends linearly with slopes of $11.12 \mathrm{pm} /{ }^{\circ} \mathrm{C}$ provided from the first experiment. The compensated slope with sensitivity of $11.33 \mathrm{pm} /{ }^{\circ} \mathrm{C}$ is in full accord with the temperature calibration. The result shows that the temperature sensor system has analogous self-compensated sensing capability by interfering with strain.

\section{Conclusions}

In this paper, an IFPI-based temperature sensor system with strain self-compensation fabricated by FS laser has been demonstrated. Experimental results indicate that temperature information is completely refined based on the FFTbased wavelength tracking method. Reconstructed spectrum of the main IFPI sensor is analyzed on the basis of temperature-strain crosstalk calculation. The result shows that these cascaded IFPI sensors have high sensitivity of temperature in $11.33 \mathrm{pm} /{ }^{\circ} \mathrm{C}$ with interference from dynamic strain variation. The sensor system also has a linear response of strain in $1.17 \mathrm{pm} / \mu \varepsilon$, provided by the compensation IFPI sensor. There is no sensing performance difference under single or multiple parameters effect. These results indicate that the presented temperature sensor system is competent with theoretical analysis in terms of the sensitivity and measurement resolution. Specific fabrication method effectively improves the quality of inscribed region and keeps the intact buffer coating of fiber, which enhance the robustness of the sensor system.

\section{Competing Interests}

The authors declare that there is no conflict of interests regarding the publication of this paper.

\section{References}

[1] Y. Zhang, J. Huang, X. Lan, L. Yuan, and H. Xiao, "Simultaneous measurement of temperature and pressure with cascaded 
extrinsic Fabry-Perot interferometer and intrinsic Fabry-Perot interferometer sensors," Optical Engineering, vol. 53, no. 6, Article ID 067101, 2014.

[2] W. Wang, D. Ding, N. Chen, F. Pang, and T. Wang, "Quasidistributed IFPI sensing system demultiplexed with FFT-based wavelength tracking method," IEEE Sensors Journal, vol. 12, no. 9, pp. 2875-2880, 2012.

[3] K. Yüksel, M. Wuilpart, and P. Mégret, "Analysis and suppression of nonlinear frequency modulation in an optical frequency-domain reflectometer," Optics Express, vol. 17, no. 7, pp. 5845-5851, 2009.

[4] Z. Chen, G. Hefferman, L. Yuan, Y. Song, and T. Wei, "Ultraweak waveguide modification with intact buffer coating using femtosecond laser pulses," IEEE Photonics Technology Letters, vol. 27, no. 16, pp. 1705-1708, 2015.

[5] T. Wei, Y. Han, Y. Li, H.-L. Tsai, and H. Xiao, “Temperatureinsensitive miniaturized fiber inline Fabry-Perot interferometer for highly sensitive refractive index measurement," Optics Express, vol. 16, no. 8, pp. 5764-5769, 2008.

[6] J. Huang, X. Lan, Y. Song, Y. Li, L. Hua, and H. Xiao, "Microwave interrogated sapphire fiber michelson interferometer for high temperature sensing," IEEE Photonics Technology Letters, vol. 27, no. 13, pp. 1398-1401, 2015.

[7] Y. Song, L. Yuan, L. Hua, and H. Xiao, "Ferrofluid-based optical fiber magnetic field sensor fabricated by femtosecond laser irradiation," in Integrated Optics: Devices, Materials, and Technologies XX, 97501U, vol. 9750 of Proceedings of SPIE, 6 pages, March 2016.

[8] L. Hua, Y. Song, J. Huang, X. Lan, Y. Li, and H. Xiao, "Microwave interrogated large core fused silica fiber Michelson interferometer for strain sensing," Applied Optics, vol. 54, no. 24, pp. 7181-7187, 2015.

[9] S. Takahashi and S. Shibata, "Thermal variation of attenuation for optical fibers," Journal of Non-Crystalline Solids, vol. 30, no. 3, pp. 359-370, 1979.

[10] D. P. Hand and P. S. Russell, "Photoinduced refractive-index changes in germanosilicate fibers," Optics Letters, vol. 15, no. 2, pp. 102-104, 1990.

[11] L. Yuan, J. Huang, X. Lan, H. Wang, L. Jiang, and H. Xiao, "All-in-fiber optofluidic sensor fabricated by femtosecond laser assisted chemical etching," Optics Letters, vol. 39, no. 8, pp. 2358-2361, 2014.

[12] E. Chmielewska, W. Urbańczyk, and W. J. Bock, "Measurement of pressure and temperature sensitivities of a Bragg grating imprinted in a highly birefringent side-hole fiber," Applied Optics, vol. 42, no. 31, pp. 6284-6291, 2003. 


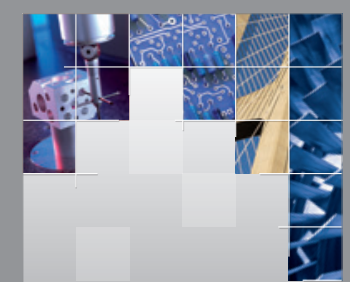

\section{Enfincering}
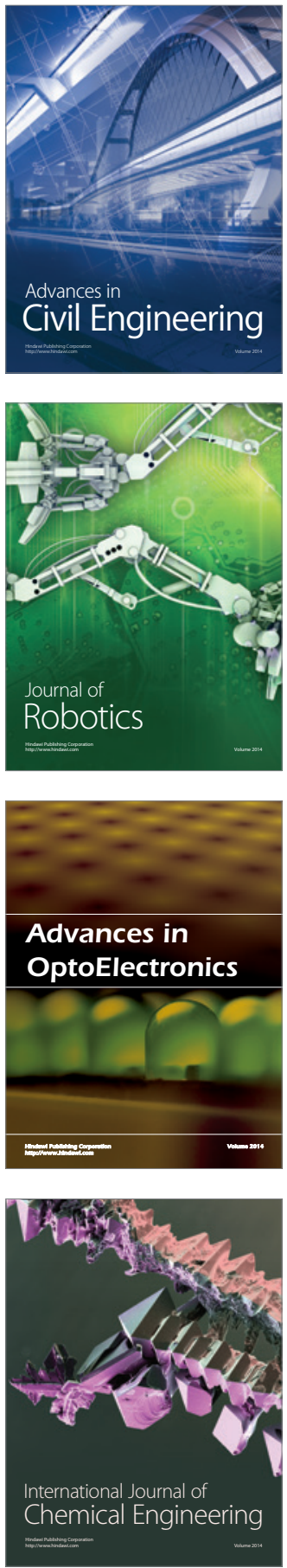

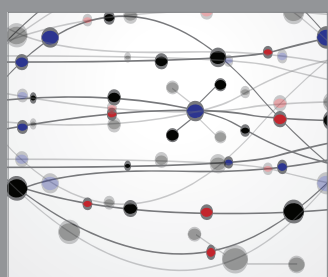

The Scientific World Journal

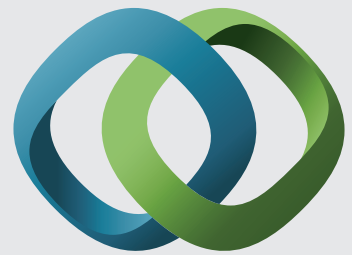

\section{Hindawi}

Submit your manuscripts at

http://www.hindawi.com
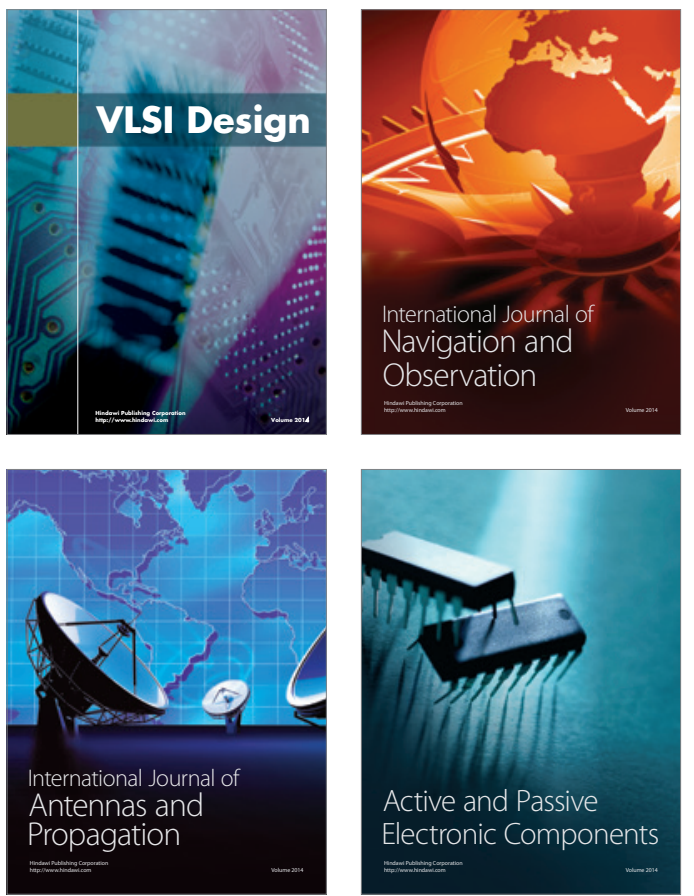
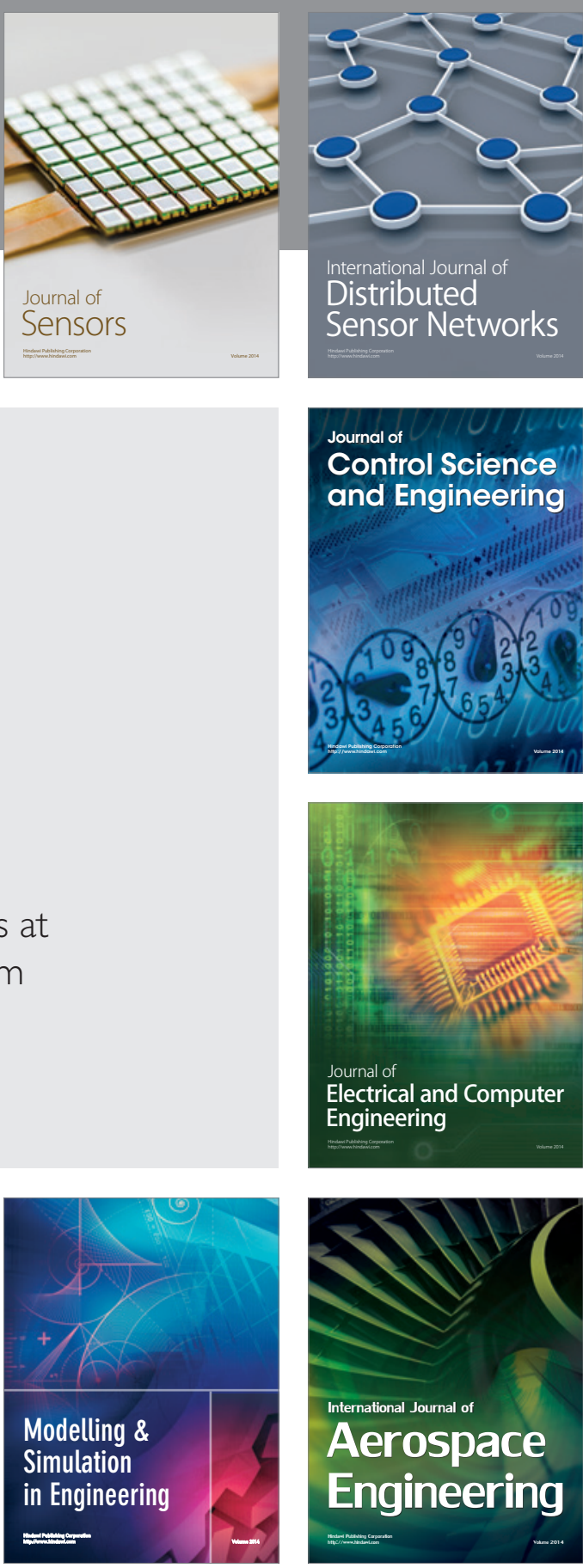

International Journal of

Distributed

Sensor Networks

Journal of

Control Science

and Engineering
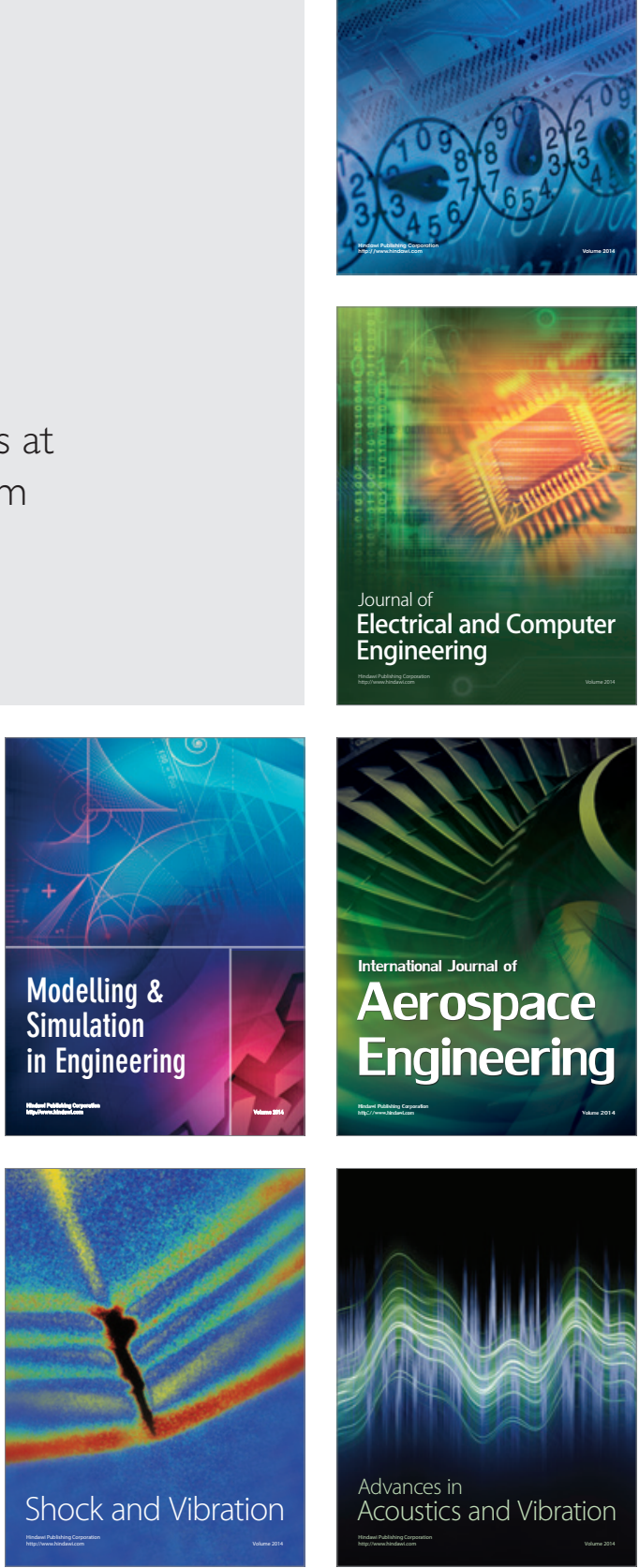